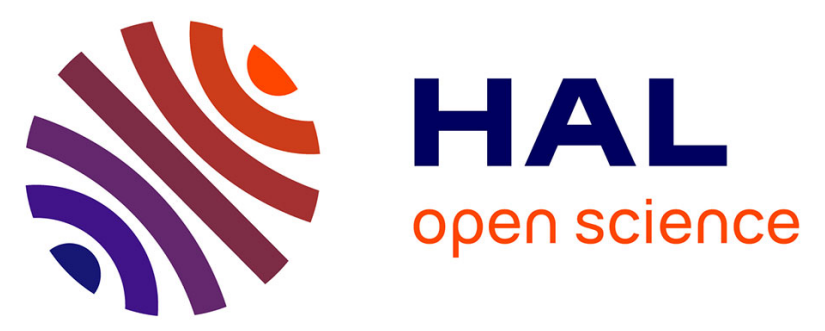

\title{
Detection of Drug-Drug Interactions Inducing Acute Kidney Injury by Electronic Health Records Mining
} Yannick Girardeau, Claire Trivin, Pierre Durieux, Christine Le Beller, Agnès Lillo-Le Louet, Antoine Neuraz, Patrice Degoulet, Paul Avillach

\section{- To cite this version:}

Yannick Girardeau, Claire Trivin, Pierre Durieux, Christine Le Beller, Agnès Lillo-Le Louet, et al.. Detection of Drug-Drug Interactions Inducing Acute Kidney Injury by Electronic Health Records Mining. Drug Safety, 2015, pp.1-11. 10.1007/s40264-015-0311-y · hal-01174261

\section{HAL Id: hal-01174261 \\ https://hal.sorbonne-universite.fr/hal-01174261}

Submitted on 8 Jul 2015

HAL is a multi-disciplinary open access archive for the deposit and dissemination of scientific research documents, whether they are published or not. The documents may come from teaching and research institutions in France or abroad, or from public or private research centers.
L'archive ouverte pluridisciplinaire HAL, est destinée au dépôt et à la diffusion de documents scientifiques de niveau recherche, publiés ou non, émanant des établissements d'enseignement et de recherche français ou étrangers, des laboratoires publics ou privés. 


\title{
Detection of Drug-Drug Interactions Inducing Acute Kidney
}

\section{Injury by Electronic Health Records Mining}

\author{
Yannick Girardeau ${ }^{1,2}$, Claire Trivin ${ }^{3}$, Pierre Durieux ${ }^{1,2}$,
}

Christine Le Beller ${ }^{4}$, Lillo-Le Louet Agnes ${ }^{4}$, Antoine Neuraz ${ }^{2}$, Patrice Degoulet ${ }^{1,2}$, Paul Avillach ${ }^{1,2,5,6}$

${ }^{1}$ Biomedical informatics and public health department. HEGP. AP-HP, Paris. France

${ }^{2}$ Sorbonne Universités, UPMC Univ Paris 06, UMR_S 1138, Centre de Recherche des Cordeliers, F75006, Paris, France

${ }^{3}$ Department of Nephrology. Hôpital Européen Georges Pompidou. Assistance Publique - Hôpitaux de Paris. Paris, France

${ }^{4}$ Department of Pharmacovigilance. Hôpital Européen Georges Pompidou. Assistance Publique Hôpitaux de Paris. Paris, France

${ }^{5}$ Center for Biomedical Informatics, Harvard Medical School, Boston, MA

${ }^{6}$ Children's Hospital Informatics Program, Boston Children's Hospital, Boston, MA

Correspondent author: Dr Paul Avillach

- Adress: Center for Biomedical Informatics 10 Shattuck Street Boston, MA 02115

- fax number:

- telephone number: +1 8578910512

- E-mail address: paul_avillach@hms.harvard.edu 


\section{ABSTRACT}

Objective: While risk of Acute Kidney Injury (AKI) is well-documented as adverse effects of some drugs, few studies have assessed relationship between Drug-Drug Interactions (DDI) and AKI. Our objective was to develop an algorithm capable of detecting potential signals on this relationship by mining retrospectively data from an electronic health record.

Material and methods: Data were extracted from the clinical datawarehouse (CDW) of the Hôpital Européen Georges Pompidou (HEGP). AKI was defined as the first level of the RIFLE criteria i.e an increase $\geq 50 \%$ of creatinine basis. Algorithm acuracy was tested on 20 single drugs, 10 nephrotoxic and 10 non-nephrotoxic. We tested then 45 pairs of nonnephrotoxic drugs, among the most prescribed at our hospital and representing distinct pharmacological classes for DDI.

Results: Sensitivity and specificity were respectively: $50 \%$ [95\% $\mathrm{Cl}, 23.66 \%-76.34 \%]$, and $90 \%$ [95\% Cl, 59.58\%-98.21\%] for single drugs. Our algorithm confirmed a precedently identified signal concerning clarithromycin and calcium-channel blockers (ORu 2.92 [95\% $\mathrm{Cl}, 1.11-7.69], P=0.04)$. Among the 45 drug pairs investigated, we identified a signal concerning 55 patients on association with bromazepam and hydroxyzine (ORu 1.66 $[95 \% \mathrm{Cl}, 1.23-2.23])$. This signal was not confirmed after a chart review. Even so, AKI and co-prescription were confirmed for respectively $96 \%$ [95\% Cl, 88\%-99\%] and $88 \%[95 \% \mathrm{Cl}$, 76\%-94\%] of these patients.

Conclusion: Data mining techniques on CDW can foster the detection of adverse drug reaction when drugs are used alone or in combination. 


\section{Key Points}

- The re-use of data from Electronic Health Record (EHR) generated by real clinical activities for pharmacoepidemiological studies is booming, it seemed that DDIs could be identified by mining such databases.

- We have developed a new signal detection algorithm using data mining techniques and the RIFLE criteria capable to re-use real care data from EHR in order to identify potential pharmacovigilance signal concerning DDIs and AKI.

- This study confirms that real care data from EHRs could be utilized to identify new pharmacovigilance signal concerning DDIs.

\section{Introduction}

Adverse Drug Reaction (ADR) can result either from the prescription of single drug or from the prescription of a combinations of drugs (CADRs) considered as a special class of Drug-Drug Interactions (DDIs). DDI, in a large sense, can also be defined as a clinically significant alteration in the effect of one drug, as a consequence of co-administration of another drug.[1] While most of the ADRs can be expected and hence can be avoided, CADRs are more highly complex to identify and, as a consequence, not well studied. CADRs could represent between 6-30\% of unexpected adverse drug effects.[2] Spontaneous Reporting Systems (SRS) are very reliable data sources for early detection of rare ADRs in post-marketing surveillance. However, at least three limitations of SRS are well known. First, most ADRs are under-reported in SRS, especially for those already known or less serious; the median under-reporting rate was estimated at $94 \%$ in a systematic review in 2006.[3] Second, some ADRs can be over-reported, especially those highlighted by media. Third, SRS do not allow the calculation of a true incidence rate in 
the lack of precise knowledge on the exact number of patients exposed to a drug. These difficulties are even greater for DDIs since the number of reports is lower. A high potential approach consists in reusing data collected and stored in Electronic Health Records (EHR), either directly or through mining a Clinical Data Warehouse (CDW) derived from an EHR. It becomes, then, possible to identify, confirm, or refute pharmacovigilance signals coming from Adverse Event Reporting System database[4,5] or eventually directly suggest new ADRs.[6-9] The combination of these methods could finally decrease the time consuming and financial cost of ADR detection for the community.[10]

Methods and tools, known as data mining, have been developed over the years to analyse large sets of data. Data mining methods in pharmacovigilance have been used with several goals, $[8,9]$ (i) in order to automate the search of publications concerning ADR in Medline,[11] (ii) to correlate and predict post-marketing adverse drug effects based on screening data from public databases of chemical structures like Pubchem,[12] (iii) to develop new algorithms to detect new or latent multi-drug adverse events in Adverse Event Reporting System database[5] and (iv) to find out new pharmacovigilance signal by mining EHR data.[13,14] For example, in a recently published work, [13] Tatonetti NP et al. confirmed a potential signal from the Food and Drug Administration's Adverse Event Reporting System, between diabetes and co-prescription of paroxetine, a selective serotonin re-uptake inhibitor and pravastatin, a cholesterol lowering agent by using data from EHRs. It was the first time that real life care data constrained in EHR were used in order to confirm a potential signal regarding DDIs.

We payed particular attention to a frequent adverse effect of drugs: Acute Kidney Injury (AKI). Nephrotoxic drugs are involved in $19-25 \%$ of the cases of severe acute renal failure in critically ill patients. $[15,16]$ Although, there is still no total consensual definition of AKI, 
the RIFLE criteria are one of the most used and well defined[17,18] and, primarily considered in our study, because of their high sensitivity and specificity and their independent association with morbidity and mortality.[19,20] Although the association between AKI and some classes of drugs (such as antiretroviral drugs, non-steroidal antiinflammatory drugs, angiotensin-converting enzyme inhibitors, aminoglycosides...) are well-known and, therefore, can be expected and, hence, avoided; to this date, only few studies have investigated the association between AKI and DDIs.[21-24]

The objective of this study was to develop a new signal detection algorithm using data mining techniques, RIFLE criteria and real care data from EHR in order to identify potential pharmacovigilance signal concerning CADRs and AKI.

\section{MATERIEL AND METHODS}

\subsection{Study Site and Settings}

The Hôpital Européen Georges Pompidou (HEGP) is a teaching hospital with 24 clinical departments and 795 beds. Since 2011, HEGP has provided his researchers with a CDW, a database that collect all information from the EHR generated by clinical activities since its opening in 2000.[25,26] Thirteen years of health data collection from 606,524 different patients, including patient's history, demographics, diagnosis, symptoms, drug treatments, clinical laboratory and image results, ICD-10 codes and fulltext inpatient and outpatient reports; all made available for research. Demographic data, drug prescriptions, lab values and ICD 10 billing codes were extracted to conduct this study.

\subsection{Study Design:}

We conducted a retrospective observational studies based on HEGP's EHR data in three steps. 
Fig.1 We chose to assess the association between AKI and the co-prescription of ten drugs, representing 45 drug pairs, among the most prescribed at the Hôpital Européen Georges Pompidou (HEGP). The pharmacovigilance unit of HEGP provided a list of 393 nephrotoxic drugs that were related to Acute Kidney Injury (AKI) at least one time in litterature (http://www.biourtox.com/Mediquick7/index.cfm). We chose drugs that were not on the list and must represent different drug classes (see electronic supplementary material 1). All relevant Data were extracted from our Electronic Health Record. For each pair of drugs, we compared two cohorts: a cohort with patients on drug 1 but not drug 2 or drug 2 but not drug 1 and a cohort with patients on both drugs.

Step one concerns the selection of eligible patients according to their clinical context.

Patients had to have at least two serum creatinine levels and one electronic prescription to be included. Patients who had been hospitalized in intensive care units were not included since these units were not on the electronic prescription system at the time of the study. We excluded patients who had an obvious cause of AKI such as patients under antineoplasic agents, patients with cardiogenic, hemorrhagic, septic, traumatic and anaphylactic shock diagnosis. We also excluded patients under dialysis since variations of creatinine could not be related to the prescription or co-prescription. We used ICD10 billing codes to define the patients to exclude.

Step two concerns the selection of patients under drug treatment. For each pair of drugs tested, three cohorts were extracted from our CDW: i) patients with co-prescription of two drugs, ii) patient prescribed drug 1 but not drug 2, iii) patient prescribed drug 2 but not drug 1. In all cases, patients could also have been on other medications. These drugs were administered orally or parenterally; topical forms were not considered. We aggregated all drugs with the same International Nonproprietary Name (INN) regardless of doses.

Step three concerns the defining of $A K I$ and drug exposure. We estimated the baseline creatinine as an outpatient serum creatinine level measured within the past three months before the prescription of drug 1 or drug 2 or both.[27,28] The treatment creatinine was defined as the highest serum creatinine value measurement within the 30 days after the 
beginning of the prescription or the co-prescription.[28] The beginning of exposure to drug 1 or 2 was defined as the first start date of prescription present in our CDW. The start of the co-prescription was defined as the first start date of drug 2 while the patient was already on drug 1 (Figure 2). Therefore, in our model, patients in each cohort had to be at least under a defined drug prescription in order to differentiate " base creatinine » and « treatment creatinine » and therefore, identify an AKI related to a drug prescription.

Fig.2 Baseline creatinine was defined as the first outpatient serum creatinine level measured within the past three months, before the prescription of drug 1 or drug 2 or both.[27,28] Baseline creatinine was compared to the treatment creatinine. The treatment creatinine was defined as the highest serum creatinine value measurement within the 30 days after the beginning of the prescription or the co-prescription. [28] The start of the co-prescription was defined as the first start date of drug 2 while the patient was already on drug 1.

We included patients regardless of which drug was prescribed first (i.e., drugs pairs 1-2 and 2-1 were processed the same way).

As we worked only with start date prescriptions, regardless of end date prescriptions, we checked that patient had a start date of the first drug within 7 days before the start date of the second drug, to be sure of a genuine co-prescription.

We created an algorithm based on the first level of the RIFLE criteria to indentify whether or not patients had or had not an AKI.[17] We considered an AKI as an increase of at least $50 \%$ from the baseline creatinine within the 30 days after the start of the prescription or the co-prescription.[20]

\subsection{Validation Tests of the Algorithm:}

In a first step, to validate our method, we tested ten drugs which were not on the list of the 393 nephrotoxic drugs (Table 1) and ten drugs that were on this list (Table 2). Selected drugs were chosen among the most prescribed during the study period and must represent different drug classes. We took patients under vitamine D oral solution as 
control, a drug that is unknown, up to this date, to induce AKI.

Then, to test our method on DDIs, we aimed to identify an association between coprescription of clarithromycin and Calcium Channel Blockers (CCB); since this association has been highlighted in a recent published study to be at risk of inducing AKI.[23] For this purpose, all HEGP prescribed CCB have been pooled in the same cohort.

\subsection{Drug-Drug Interaction Concerned Two by Two Combined Drugs Administration and}

AKI:

We chose to assess the association between AKI and all 45-drug pairs combined with ten drugs unknown, up to this date, to induce AKI and picked among the most prescribed at HEGP (see electronic supplementary material 1) for the time period of the study. The 10 drugs we selected must not be on the 393 nephrotoxic drug list and must represent different drug classes.

\subsection{Chart Review Method:}

Two senior physicians from the pharmacovigilance department conducted a chart review for patients under drug pairs for which we had a signal. For each patient record, pharmacologist experts had to confirm (i) the existence of an AKI (ii) the existence of a genuine co-prescription (iii) and to find out other causes of AKI as nephrotoxic drug coprescriptions, injection of iodinated contrast media, multiple organ failure and so forth. A nephrologist was in charge to analyse unobvious cases.

\subsection{Additional Analysis:}

We conducted several additional analysis for the drug pairs for which we had a signal: (i) the impact of the prescription order, (ii) different time intervals ( 3,5 and 7 days) between the sart of co-prescription and AKI and (iii) the mean of concomitant nephrotoxic prescriptions per patient between patients under co-presciption and patients under 
single drugs.

\subsection{Statistical Methods:}

For the validation test, we estimate sensitivity, specificity, predictive positive value, predictive negative value and the Yule's $Q$ contingency coefficient of the algorithm.

Then, we conducted a logistic regression model to evaluate the asssociation between AKI and co-prescription. For this purpose, we compared two cohorts (Figure 2): a cohort with people who had drug 1 but not drug 2 or drug 2 but not drug 1 and a cohort with people on both drugs, assuming that patients under one or the other drug would be closer in terms of potential counfounding factors vs. patients with any other drugs.

In a first step, we tested the association with AKI for each pair of drugs by a Chi-squared test. The aim was to ensure that the possible association was well related to the coprescription and not to the effect of potential confounding factors specifically associated with one of the drugs, for we previously pooled patients under drug 1 or 2 in a single cohort. If there was a significant difference between the two drugs, we would abort our investigations (see electronic supplementary material 2). For each patient, age, sex, number of prescriptions at the maximum creatinine level, baseline creatinine and time period between the start date of (co-) prescription were used as covariates. Age, number of prescriptions, baseline creatinine and time period were coded as continuous variables, others as binary.

The Logistic Regression Model was Defined as follows:

Logit $($ IRA $)=$ b0 +b1Drug1\&2/Drug1xor2 + b2Age + b3Sex + b4Baseline_creatinine + b5Number of precriptions + b6Time_period

The number of prescriptions, at the date of the treatment creatinine, ignored saline, glucose infusions and topical form medications. 
An Open Database Connection (ODBC) linking an Oracle ${ }^{\circledR}$ database (11g Enterprise Edition

Release 11.2.0.1.0) of $\mathrm{i} 2 \mathrm{~b} 2 \mathrm{CDW}$ (version 1.3) to R software (version 2.15.3) was set up. The extraction of data was performed with SQL queries then R was used for all the analysis (RODBC, stats, MASS and epitools Packages). We used the false discovery rate control method to correct multiple comparisons.

We obtained an approval from the institutionnal review board of our hospital (IRB\#00001072 Study \#CDW_2013_0004).

\section{RESULTS}

\subsection{Data mining and clinical screening of patients cohorts (Figure 3):}

We extracted data from 606,524 patients from July 2000 to June 2013 from HEGP CDW.[25,26] Off these patients, 88,687 patients had at least two serum creatinine levels and one electronic prescription. We excluded patients who had obvious cause of creatinine variation i.e., 12,620 patients with at least one ICD-10 billing codes for shocks or antineoplastic agents or dialysis. Finally, 76,067 patients had been included in this study.

Fig.3 Flow diagram: we extracted data from the Clinical Data Warehouse (CDW) of the Hôpital Européen Georges Pompidou (HEGP) containing care data of 606,524 patients from July 2000 to June 2013. 88,687 patients had at least two serum creatinine levels and one electronic prescription. Out of these patients, 12,620 had at least an ICD-10 billing codes of shocks or antineoplastic agents or dialysis and were excluded. Finally, 76,067 patients had been included in this study.

\subsection{Testing our algorithm on pairwise single drugs-AKI:}

The validation of the algorithm, for AKI adverse event detection, was performed on 20 types of single prescription drugs among the most prescribed in our hospital: (i) 10 drugs 
known to cause AKI; (ii) 10 drugs not known to cause AKI. Vitamine D oral solution was considered as control.

\subsubsection{Acute Kidney Injury and Non Nephrotoxic Drugs:}

Table 1: Testing our Signal Identification Algorithm on ten Non Nephrotoxic Drugs.

\begin{tabular}{|c|c|c|c|c|c|}
\hline Tested Drugs & $\begin{array}{c}\text { Drug } \\
\text { but not VIT-D } \\
\text { N (AKI/ NO AKI) }\end{array}$ & $\begin{array}{c}\text { VIT-D } \\
\text { but not drug } \\
\text { N (AKI/ NO AKI) }\end{array}$ & ORu & $P$ value & $\begin{array}{l}P \text { value } \\
\text { corrected }\end{array}$ \\
\hline ACETYLCYSTEINE & $110 / 1,489$ & $40 / 744$ & $\begin{array}{c}1.37 \\
{[0.94 ; 1.99]}\end{array}$ & 0.106 & 0.278 \\
\hline ATENOLOL & $58 / 971$ & $54 / 823$ & $\begin{array}{c}0.91 \\
{[0.62 ; 1.33]}\end{array}$ & 0.696 & 0.875 \\
\hline FOLIC ACID & 75/998 & $49 / 764$ & $\begin{array}{c}1.17 \\
{[0.81 ; 1.70]}\end{array}$ & 0.453 & 0.850 \\
\hline GLIMEPIRIDE & $27 / 401$ & $52 / 820$ & $\begin{array}{c}1.06 \\
{[0.65 ; 1.71]}\end{array}$ & 0.806 & 0.896 \\
\hline LOPERAMIDE & $70 / 710$ & $49 / 803$ & $\begin{array}{c}1.62 \\
{[1.11 ; 2.36]}\end{array}$ & 0.013 & 0.066 \\
\hline METOPROLOL & $58 / 971$ & $55 / 822$ & $\begin{array}{c}0.91 \\
{[0.62 ; 1.33]}\end{array}$ & 0.70 & 0.875 \\
\hline MIANSERIN & $39 / 433$ & $50 / 790$ & $\begin{array}{c}1.42 \\
{[0.92 ; 2.22]}\end{array}$ & 0.111 & 0.278 \\
\hline $\begin{array}{l}\text { PHYSIOLOGICAL } \\
\text { SALINE 0,5L \& } 1 \text { L }\end{array}$ & $550 / 6,203$ & $17 / 380$ & $\begin{array}{c}1.98 \\
{[1.21 ; 3.25]}\end{array}$ & 0.004 & 0.040 \\
\hline TAMSULOSIN & $85 / 1,221$ & $50 / 738$ & $\begin{array}{c}1.03 \\
{[0.72 ; 1.47]}\end{array}$ & 0.927 & 0.927 \\
\hline TRIMETAZIDINE & $24 / 301$ & $55 / 826$ & $\begin{array}{c}1.20 \\
{[0.73 ; 1.97]}\end{array}$ & 0.51 & 0.850 \\
\hline $\begin{array}{l}\text { VITAMINE D } \\
\text { Oral solution }\end{array}$ & 0 & 901 & 1 & - & - \\
\hline
\end{tabular}

ORu, unadjusted Odds Ratio

Among the 10 drugs not known to cause AKI, we didn't find any association with AKI for all drugs except for physiological saline (odds ratio (OR) 1.98 [95\% Cl. 1.21-3.25], $P$ corrected $=0.040)($ Table 1$)$ after multiple testing correction .

\subsubsection{Acute Kidney Injury and Nephrotoxic Drugs:}

Among the 10 drugs known to cause AKI, we found a statistically significant association with AKI for 5 of them after multiple testing correction. (Patients under amphotericine B (ORu 2.79 [95\%Cl, 1.87-4.16], absolute risk increase (ARI) 7.09\% [95\%Cl, 4.49\%-9.73\%], 
number needed to harm (NNH) 14), patients under ciprofloxacin (ORu $1.82[95 \% \mathrm{Cl}, 1.27-$ 2.61], ARI 4.39\% [95\%Cl, 1.77\%-7.04\%], NNH 23), patients under gentamicin (ORu 2.96 [95\%Cl, 2.11-4.15], ARI 9.07\% $[95 \% \mathrm{Cl}, 6.55 \%-11.48 \%], \mathrm{NNH} 11)$, patients under metronidazole (ORu 1.97 [95\%Cl, 1.36-2.86], ARI 4.68\% [95\%Cl, 2.18\%-7.19\%], NNH 21) and patients under vancomycin (ORu $2.23[95 \% \mathrm{Cl}, 1.57-3.16], \mathrm{ARI} 5.90 \%[95 \% \mathrm{Cl}, 3.49 \%-$ 8.21\%], NNH 17) (Table 2).

Table 2: Testing our Signal Identification Algorithm on ten Nephrotoxic Drugs.

\begin{tabular}{|c|c|c|c|c|c|}
\hline Tested Drugs & $\begin{array}{c}\text { Drug } \\
\text { but not VIT-D } \\
\text { N (AKI/ NO AKI) }\end{array}$ & $\begin{array}{c}\text { VIT-D } \\
\text { but not drug } \\
\text { N (AKI/ NO } \\
\text { AKI) }\end{array}$ & ORu & $P$ value & $\begin{array}{c}P \text { value } \\
\text { corrected }\end{array}$ \\
\hline ALLOPURINOL & $114 / 1,300$ & $51 / 779$ & $\begin{array}{c}1.34 \\
{[0.95 ; 1.89]}\end{array}$ & 0.0946 & 0.158 \\
\hline AMPHOTERICINE B & $98 / 749$ & $35 / 747$ & $\begin{array}{c}2.79 \\
{[1.87 ; 4.16]}\end{array}$ & $1.63 e-07$ & $8.15 e-07$ \\
\hline CAPTOPRIL & $22 / 292$ & $55 / 840$ & $\begin{array}{c}1.15 \\
{[0.69 ; 1.92]}\end{array}$ & 0.592 & 0.592 \\
\hline CIPROFLOXACIN & $87 / 752$ & $50 / 786$ & $\begin{array}{c}1.82 \\
{[1.27 ; 2.61]}\end{array}$ & $1.26 \mathrm{e}-03$ & $2.52 \mathrm{e}-03$ \\
\hline GENTAMICIN & $190 / 1,122$ & $45 / 786$ & $\begin{array}{c}2.96 \\
{[2.11 ; 4.15]}\end{array}$ & $1.37 e-11$ & $1.37 e-10$ \\
\hline KETOPROFEN & $27 / 471$ & $55 / 826$ & $\begin{array}{c}0.86 \\
{[0.53 ; 1.38]}\end{array}$ & 0.556 & 0.592 \\
\hline METFORMIN & $77 / 1,453$ & $50 / 772$ & $\begin{array}{c}0.94 \\
{[0.57 ; 1.18]}\end{array}$ & 0.293 & 0.367 \\
\hline METRONIDAZOLE & $92 / 824$ & $44 / 777$ & $\begin{array}{c}1.97 \\
{[1.36 ; 2.86]}\end{array}$ & $3.19 e-04$ & 7.97e-04 \\
\hline RIFAMPIN & $25 / 255$ & $53 / 820$ & $\begin{array}{c}1.46 \\
{[0.88 ; 2.41]}\end{array}$ & 0.168 & 0.240 \\
\hline VANCOMYCIN & $143 / 1,121$ & $44 / 769$ & $\begin{array}{c}2.23 \\
{[1.57 ; 3.16]}\end{array}$ & $3.03 e-06$ & $1.01 \mathrm{e}-05$ \\
\hline $\begin{array}{l}\text { VITAMINE D } \\
\text { Oral solution }\end{array}$ & 0 & 901 & 1 & - & - \\
\hline
\end{tabular}

\subsubsection{Acuracy of our algorithm:}

We evaluated the sensitivity and specificity of our method considering identified drugs in table 1 as false positive and, identified drugs in table 2 as true positive (Se $50 \%[95 \% \mathrm{Cl}$, 23.66\%-76.34\%], Sp 90\% [95\%Cl. 59.58\%-98.21\%]. Positive likelihood ratio (PLR) were 5 
$[95 \% \mathrm{Cl}, 0.7-35.5]$ and negative likelihood ratio $(\mathrm{NLR})$ were $0.6 \%[95 \% \mathrm{Cl} .0 .3-1.1]$ using correction for multiple test. The Yule's $Q$ contingency coefficient indicated a strong association 0.71 .

\subsection{Acute kidney injury and drug-drug interactions:}

To assess our method on DDIs, we first tested a pair of drugs that was already known to be associated with AKI : clarithromycin and calcium-channel blockers.[23] We found a statistically significant association between AKI and this co-prescription by re-using our health care data (ORu $2.92[95 \% \mathrm{Cl}, 1.11-7.69] P=0.04)$.

To identify new pharmacovigilance signal concerning DDIs, we investigated forty-five pairs of drugs that could be formed with ten drugs we selected among the most prescribed at HEGP (see electronic supplementary material 1). Only 27 pairs of drugs were eligble for comparison after a Chi square test (see methods \& see electronic supplementary material 2). Considering unadjusted Odds Ratio, the co-prescription of one pair of drugs was associated with AKI: hydroxyzine and bromazepam (ORu 1.66 [95\%Cl, 1.23-2.23], ARI 3.70\% [95\%Cl, 1.39\%-6.52\%], NNH 27).

Table 3: Association between Acute Kidney Injury and Drug Drug Interaction for 27 Comparable Drug Pairs.

\begin{tabular}{|lcccc|}
\hline DRUG PAIRS (Drug 1/ Drug 2) & $\begin{array}{c}\text { Cohort } \\
\text { Drug } 1 \text { or } 2 \\
\text { N (AKI/NO AKI) }\end{array}$ & $\begin{array}{c}\text { Cohort } \\
\text { Drug } 1 \& 2 \\
\text { N (AKI/NO AKI) }\end{array}$ & ORu & $\begin{array}{c}P \\
\text { corrected }\end{array}$ \\
HYDROXYZINE/BROMAZEPAM & $352 / 5,266$ & $56 / 506$ & $1.66[1.23 ; 2.23]$ & 0.04 \\
NICARDIPINE/BISOPROLOL & $482 / 6,714$ & $50 / 440$ & $1.58[1.16 ; 2.15]$ & 0.07 \\
PHLOROGLUCINOL/NICARDIPINE & $336 / 4,111$ & $32 / 231$ & $1.69[1.15 ; 2.49]$ & 0.11 \\
CONTRAMAL/NICARDIPINE & $525 / 7,851$ & $87 / 990$ & $1.31[1.04 ; 1.66]$ & 0.17 \\
ACUPAN/BISOPROLOL & $550 / 8,106$ & $50 / 531$ & $1.39[1.03 ; 1.89]$ & 0.20 \\
BROMAZEPAM/BISOPROLOL & $417 / 6,262$ & $59 / 675$ & $1.31[0.99 ; 1.74]$ & 0.30 \\
PHLOROGLUCINOL/BROMAZEPAM & $347 / 4,173$ & $28 / 238$ & $1.41[0.94 ; 2.12]$ & 0.38 \\
ACUPAN/HYDROXYZINE & $388 / 5,799$ & $72 / 929$ & $1.16[0.89 ; 1.50]$ & 0.56 \\
L-THYROXINE/MACROGOL & $394 / 5,876$ & $24 / 447$ & $0.80[0.52 ; 1.22]$ & 0.56 \\
HYDROXYZINE/BISOPROLOL & $496 / 7,426$ & $67 / 848$ & $1.18[0.91 ; 1.54]$ & 0.56 \\
NICARDIPINE/BROMAZEPAM & $357 / 4,326$ & $26 / 251$ & $1.26[0.83 ; 1.91]$ & 0.56 \\
CONTRAMAL/BROMAZEPAM & $549 / 8,335$ & $60 / 780$ & $1.17[0.89 ; 1.54]$ & 0.56 \\
NICARDIPINE/ACENOCOUMAROL & $310 / 3,669$ & $7 / 128$ & $0.65[0.30 ; 1.40]$ & 0.56 \\
ACUPAN/BROMAZEPAM & $407 / 5,473$ & $28 / 473$ & $0.80[0.54 ; 1.18]$ & 0.56 \\
ACUPAN/NICARDIPINE & $406 / 5,274$ & $51 / 547$ & $1.21[0.89 ; 1.64]$ & 0.56 \\
\hline
\end{tabular}




\begin{tabular}{|lcccc|}
\hline CONTRAMAL/HYDROXYZINE & $558 / 8,450$ & $94 / 1,280$ & $1.11[0.89 ; 1.39]$ & 0.56 \\
HYDROXYZINE/NICARDIPINE & $431 / 5,472$ & $27 / 422$ & $0.81[0.54 ; 1.21]$ & 0.56 \\
PHLOROGLUCINOL/ACENOCOUMAROL & $276 / 3,819$ & $28 / 262$ & $0.54[0.87 ; 1.53]$ & 0.56 \\
ACUPAN/ACENOCOUMAROL & $399 / 5,380$ & $16 / 154$ & $1.40[0.83 ; 2.37]$ & 0.58 \\
HYDROXYZINE/ACENOCOUMAROL & $366 / 5,223$ & $17 / 211$ & $1.15[0.69 ; 1.91]$ & 0.75 \\
L-THYROXINE/ACUPAN & $413 / 5,489$ & $17 / 272$ & $0.83[0.50 ; 1.37]$ & 0.75 \\
ACENOCOUMAROL/BISOPROLOL & $404 / 5,819$ & $33 / 505$ & $0.94[0.65 ; 1.36]$ & 0.95 \\
CONTRAMAL/ACENOCOUMAROL & $590 / 8,545$ & $28 / 388$ & $1.05[0.71 ; 1.55]$ & 0.95 \\
CONTRAMAL/BISOPROLOL & $622 / 9,863$ & $85 / 1,368$ & $0.99[0.78 ; 1.24]$ & 0.95 \\
BROMAZEPAM/ACENOCOUMAROL & $263 / 3,429$ & $20 / 250$ & $1.04[0.65 ; 1.67]$ & 0.95 \\
L-THYROXINE/CONTRAMAL & $584 / 8,635$ & $36 / 507$ & $1.05[0.74 ; 1.49]$ & 0.95 \\
CONTRAMAL/ACUPAN & $468 / 7,053$ & $142 / 2,116$ & $1.01[0.83 ; 1.23]$ & 0.95 \\
\hline
\end{tabular}

\subsection{Study of Covariates:}

We conducted a covariates study for people under hydroxyzine and/nor bromazepam.

We tested age, sex, base creatinine, number of concomitant prescription and time period to AKI. (Table 4) Three covariates were significantly associated with AKI in a univariate analysis, age $(P=6.93 \mathrm{e}-06)$, number of concomitant prescriptions $(P=1.01 \mathrm{e}-07)$ and time period $(P<2 \mathrm{e}-16)$. OR adjusted for these 3 covariates was: 1.47 [95\% $\mathrm{Cl}, 1.07-1.99]$.

Table 4: Demographic and Covariates Characteristics for (i) Hydroxyzine and Bromazepam, (ii) Hydroxyzine XOR Bromazepam Cohort

\begin{tabular}{|lcc|}
\hline & $\begin{array}{c}\text { Hydroxyzine } \\
\text { XOR }\end{array}$ & $\begin{array}{c}\text { Hydroxyzine } \\
\text { ANomazepam } \\
\text { Bromazepam }\end{array}$ \\
Age (mean \pm SD) & $62 \pm 19$ & $60 \pm 18$ \\
Sex (\%female) & 45 & 49 \\
Base creatinine (mean \pm SD) & $97 \pm 65$ & $101 \pm 78$ \\
Comedications (N $\pm S D)$ & $7 \pm 5.5$ & $8.6 \pm 6.5$ \\
Time period to AKI (Median[1Q,30]) & $2.4[1.2,6.4]$ & $2.5[1.3,7.3]$ \\
\hline
\end{tabular}




\subsection{Testing of a Potential Class Effect:}

Four other benzodiazepines were prescribed at HEGP: alprazolam, diazepam, lorazepam and oxazepam. We tested each one of them in association with hydroxyzine but did not find any significative association with AKI after multiple testing correction ( $P$ was respectively $1,0.72,1,0.24)$. The result was equivalent when we pooled patients with all benzodiazepines but bromazepam $(P=0.68)$. We only tested for hydroxyzine as it is the unique representant of its ATC drug class.

\subsection{Additional Analysis:}

We tested the impact of the prescription order and found out a difference: the association was present when bromazepam was prescribed first $(1.77$ [95\% Cl, 1.24-2.54], $P=0.003)$ but not when hydroxyzine was also first $(1.21[95 \% \mathrm{Cl}, 0.73-2.00], P=0.49)$. Then, we tested association between AKI and hydroxyzine and bromazepam concomitant prescription for different time intervals: 3,5 and 7 days between the start of coprescription and serum creatinine levels of interest and did not find any association, $P$ were $0.44,0.16$ and 0.13 respectively. We tested the assumption that nephrotoxic drugs will be equally distributed between prescription and co-prescription cohorts. The mean of concomitant nephrotoxic prescriptions was not statistically different between patients under hydroxyzine or bromazepam alone (mean $=5.76$ ) and patients under both drugs $($ mean $=4.82)(P=0.089)$.

\subsection{Chart Review:}

To further our investigations on the potential signal concerning hydroxyzine and bromazepam, 56 patient's records who were under this co-prescription and had an AKI were reviewed by two senior physicians from the pharmacovigilance department. The diagnoses of AKI had been confirmed for $96 \%$ [95\% Cl, 88\%-99\%] of cases ( $\mathrm{N}=54)$; in one 
case, the baseline creatine level was artificially low due to an hyperhydratation, this elevation of creatinine was in fact a normalisation. The concomitant prescription was confirmed for $88 \%$ [95\%Cl, $76 \%-94 \%$ ] of cases $(\mathrm{N}=49)$. All patients had another cause of AKI: renal causes, including functional renal failure $(N=20)$, other drugs $(N=15)$, mixed causes (clinical status, drugs and functional renal failure) $(N=15)$, infection $(N=3)$. A nephrologist analysed three unobvious cases that were finaly classified in mixed causes.

\section{DISCUSSION}

Identification of new DDIs is a major public health concern. While SRS is not suitable for the identification of new DDIs, the secondary use of clinical data, collected in EHRs to identify new signals for DDIs is promising. We created an algorithm capable of extracting EHR's data and identify association between concomitant prescriptions and AKI. AKI was identified using real laboratory data and defined by the first level of RIFLE criteria for each patient and each drug and drug pair tested.[18] As ADR of single drugs are better known, we tested first our algorithm on single drug pairs and were able to identify signals concerning drugs known to be nephrotoxic with quite good accuracy (sensitivity: 50\% [95\%Cl, 23.66\%-76.34\%], specificity: $90 \%$ [95\%Cl, 59.58\%-98.21\%]). The lack of sensitivity could have resulted from an indication bias since nephrotoxic drugs are prescribed very carefully to people with poor kidney function. However, such exploratory studies often suffer from a lack of specificity induced by the oversize number of false positive signals. Increasing the sensitivity of the method could have resulted in a lack of specificity which was not desirable for the second step of the study.

In order to test our algorithm on DDIs, we investigated clarithromycin and CCB concomitant prescriptions which were associated recently with higher risk of hospitalization with AKI (OR $=1.98[95 \% \mathrm{Cl}, 1.68-2.34]) \cdot[23]$ We identified a significant 
statistical association between co-prescription of these drugs and AKI, ORu $2.92[95 \% \mathrm{Cl}$, 1.11-7.69], $P=0.04)$. By investigating all drug pairs formed with ten drugs chosen among the most prescribed in our hospital, we identified a potential signal linked co-medication of hydroxyzine and bromazepam and AKI (ORu 2.23 [95\%Cl, 1.57-3.16], ORa 1.47 [95\%Cl, 1.07-1.99]). This signal was not confirmed by a class effect study, different onset time and a chart review. Although, other plausible causes of AKI have been detected for all patients, chart review confirmed an effective AKI in $98 \%[95 \% \mathrm{Cl}, 88 \%-99 \%]$ of cases and, a concomitant prescriptions in $88 \%$ [95\% Cl, 76\%-94\%] of cases. For pharmacovigilance, these results are extremely encouraging, in the aim of the secondary used of real world care data contained in EHR, whose main purpose of the present study was not to formally identify a relationship between tested co-prescriptions and AKI but, to provide a new tool for the identification of potential cases for pharmacovigilance hospital units. This work could be the first step of further studies as the detection of DDIs concerning comedications of more than two drugs, detection of over DDIs or to develop EHRs alarm systems for drugs that have been recently marketed.

\subsection{Start Date of Therapy and Beginning of Exposure:}

As the only source of data used came from our hospital database, we had no information regarding prescription outside the hospital. Thus, if the first prescription occurred before hospitalization, the beginning of exposure could be potentially confused with a simple renewal of treatment. To reduce this bias, we should have had manually analysed each patient's record to trace off a previous exposure before the hospitalization. It would have been possible to analyse a few drug pairs but not in a data mining perspective which is to analyse the largest possible number of drug pairs as possible. This bias has already been 
discused for such studies but it would tend to minimize the signal and therefore reduce the detection capability of the algorithm.[13]

\subsection{Concomitant Nephrotoxic Prescriptions:}

In all studied cases, patients of each cohort could be on other prescription. In a first analysis, we tried to exclude patients on concomitant nephrotoxic treatment from the the 393 nephrotoxic drug list. Unfortunately, the lost of many patients made the analysis no longer feasible. We finally chose to keep concomitant potential nephrotoxic drugs as we accepted the hypothesis that they should be equaly distributed in each cohort.

This hypothesis was reasonable since there was no reason to think that a nephrotoxic drug could be associated to a co-prescription rather than the single medications tested. Furthermore, there were no significant difference $(P=0.089)$ between the mean of concomitant nephrotoxic drug prescriptions per patient in the cohorts of hydroxyzine or bromazepam alone and hydroxyzine and bromazepam in association.

\section{3 lodinated Contrast Media:}

AKI is a classical adverse effect of iodinated contrast media. Unfortunately, we were not able to exclude patient who underwent an injection of iodinated contrast media as their prescriptions not recorded in our CDW. We accepted similar hypothesis with concomitant prescription of nephrotoxic drugs. There was no reason to think that there were more patients under iodinated contrast media in the co-prescription cohort than in the single prescription cohort.

\subsection{Limitation of Data Mining in Retrospective Observational Study:}

Our objective was to analyze the largest possible number of drug pairs. Hence, we could not achieve a traditionnal covariate analaysis. For exploratory purposes, we restricted our analysis to five relevant factors: age, sex, baseline creatinine, number of concomitant 
prescription and time period between start date of prescription and max creatinine level.[20] We choose to test our covariates only for relevent drug pairs in order to avoid over-adjustment bias. Moreover, in this first phase of the analysis, a comprehensive analysis of all the potential confounding factors would have introduced too much complexity for a proper interpretation.

\subsection{Acute Kidney Injury and Serum Creatinine Base Definitions:}

We chose to work with the RIFLE classification as it is currently one of the most consensual for epidemiologic research.[17,18] These criteria can detect AKI with high sensitivity and specificity and are independently associated with morbidity and mortality. $[19,20]$ One of the limitations of this classification consists in the definition of the baseline serum creatinine level. Few definitions have been proposed in litterature and all resulted in misclassifications.[20] Baseline serum creatinine level should be representative of the normal renal function; an outpatient serum creatinine level measured within the past 3 months have been proposed by Ricci et al. in 2011.[18] We chose to work with the nearest outpatient serum creatinine level before the beginning of prescription or co-prescription in a 3-month timeframe. The relevance of this choice was confirmed by the chart review results, where $98 \%$ of the relevant cases had a confirmed AKI.

\subsection{Time Period Between the Inclusion and the End of Follow-up:}

Definition of a timeframe within which the increase of serum creatinine occurs is another issue of the RIFLE classification. The Acute Dialysis Quality Initiative (ADQI)[17] have chosen a one-week window; but some patients, with slowly progressive AKI, could be misidentified with this too short timeframe.[20] The onset time of acute renal failure 
following a drug prescription is highly variable from one drug to another; it goes from one hour for nifedipine - a CCB - to thirty years for lithium - a mood stabilizer (http/::www.biourtox.com:Mediquick7:index.cfm). We have retained a thirty-day window that seemed a sufficient amount of time to allow acute renal failure to occur and short enough to limit various confounding factors. This arbitrary choice should not influenced the results since the mediane of the time period between (co-)prescription and the max creatinine level was 2.4 days for hydroxyzine or bromazepam and 2.5 days for the combination cohort.

\subsection{Patient Selection and Prescription:}

The selection of a particular drug causes the selection of a particular patient. Hence, antihypertensive agents select hypertensive patients, hypoglycemic drugs select diabetics and patients on both drugs will be at greater risk to have a diabetic nephropathy and to develop an episode of acute renal failure. Thus, our results show that patients receiving physiological saline were more at risk to develop an episode of acute renal failure than patients receiving folic acid. An assumption could be that patients requiring perfusion are weaker than patients who do not and are therefore more likely to develop AKI during their hospitalization. Similarly, loperamide, an opioid drug used against diarrhea was closed to trigger a signal; hypovolemia caused by gastrointestinal fluid losses could be an explanation to this outcome. This selection bias have to be questionned for every pair of drugs and it is the problem of any epidemiological study, namely the existence of a causal relationship.

\section{CONCLUSION}

We developed a new datamining algorithm capable of detecting potential signals 
concerning DDIs and AKI by mining data from an electronic health record. Therefore, we confirmed a precedently association concerning concomitant prescription of clarithromycin and calcium-channel blockers and AKI. This work confirm the EHR's data re-using potential in a pharmacovigilance perspective.

Acknowledgements: Cendrine Baudoin, Eric Zapletal, Antoine Rachas, Abdel-Ali Boussadi, Bastien Rance, Anne-Sophie Jannot, Jean Bouyer, Gilles Chatellier.

\section{Competing Interests:}

The authors declared no conflict of interest.

\section{Ethical standards:}

All persons gave their informed consent prior to their inclusion in the study. We obtained an approval from the institutionnal review board of our hospital (IRB\#00001072 Study \#CDW_2013_0004).

\section{References}

1. Hines LE, Murphy JE. Potentially harmful drug-drug interactions in the elderly: a review. Am J Geriatr Pharmacother. 2011;9:364-77.

2. Pimohamed M. Drug interactions of clinical importance. Chapman \& Hall: London; 1998.

3. Hazell L, Shakir SAW. Under-reporting of adverse drug reactions : a systematic review. Drug Saf. 2006;29:385-96.

4. Thakrar BT, Grundschober SB, Doessegger L. Detecting signals of drug-drug 
interactions in a spontaneous reports database. Br J Clin Pharmacol. 2007;64:489-95.

5. Tatonetti NP, Fernald GH, Altman RB. A novel signal detection algorithm for identifying hidden drug-drug interactions in adverse event reports. J Am Med Inform Assoc. 2012;19:79-85.

6. Curtis JR, Cheng H, Delzell E, Fram D, Kilgore M, Saag K, et al. Adaptation of Bayesian Data Mining Algorithms to Longitudinal Claims Data: Coxib Safety as an Example. Medical Care. 2008;46:969-75.

7. Wang X, Hripcsak G, Markatou M, Friedman C. Active Computerized Pharmacovigilance Using Natural Language Processing, Statistics, and Electronic Health Records: A Feasibility Study. Journal of the American Medical Informatics Association. 2009;16:328-37.

8. Svanström H, Callréus T, Hviid A. Temporal data mining for adverse events following immunization in nationwide Danish healthcare databases. Drug Saf. 2010;33:1015-25.

9. Schuemie MJ. Methods for drug safety signal detection in longitudinal observational databases: LGPS and LEOPARD. Pharmacoepidem. Drug Safe. 2011;20:292-9.

10. Coloma PM, Trifirò G, Patadia V, Sturkenboom M. Postmarketing safety surveillance : where does signal detection using electronic healthcare records fit into the big picture? Drug Saf. 2013;36:183-97.

11. Avillach P, Dufour J-C, Diallo G, Salvo F, Joubert M, Thiessard F, et al. Design and validation of an automated method to detect known adverse drug reactions in MEDLINE: a contribution from the EU-ADR project. J Am Med Inform Assoc [Internet]. 2012 [cited 2013 May 5]; Available from: http://jamia.bmj.com/content/early/2012/11/28/amiajnl2012-001083

12. Pouliot Y, Chiang AP, Butte AJ. Predicting adverse drug reactions using publicly available PubChem BioAssay data. Clin. Pharmacol. Ther. 2011;90:90-9. 
13. Tatonetti NP, Denny JC, Murphy SN, Fernald GH, Krishnan G, Castro V, et al. Detecting Drug Interactions From Adverse-Event Reports: Interaction Between Paroxetine and Pravastatin Increases Blood Glucose Levels. Clinical Pharmacology \& Therapeutics. 2011;90:133-42.

14. Takahashi Y, Nishida Y, Nakayama T, Asai S. Comparative effect of clopidogrel and aspirin versus aspirin alone on laboratory parameters: a retrospective, observational, cohort study. Cardiovasc Diabetol. 2013;12:87.

15. Mehta RL, Pascual MT, Soroko S, Savage BR, Himmelfarb J, Ikizler TA, et al. Spectrum of acute renal failure in the intensive care unit: the PICARD experience. Kidney Int. 2004;66:1613-21.

16. Uchino S, Kellum JA, Bellomo R, Doig GS, Morimatsu H, Morgera S, et al. Acute renal failure in critically ill patients: a multinational, multicenter study. JAMA. 2005;294:813-8.

17. Bellomo R, Ronco C, Kellum JA, Mehta RL, Palevsky P. Acute renal failure - definition, outcome measures, animal models, fluid therapy and information technology needs: the Second International Consensus Conference of the Acute Dialysis Quality Initiative (ADQI) Group. Crit Care. 2004;8:R204-12.

18. Ricci Z, Cruz DN, Ronco C. Classification and staging of acute kidney injury: beyond the RIFLE and AKIN criteria. Nature Reviews Nephrology. 2011;7:201-8.

19. Ricci Z, Cruz D, Ronco C. The RIFLE criteria and mortality in acute kidney injury: A systematic review. Kidney Int. 2007;73:538-46.

20. Ostermann M, Chang RWS. Challenges of defining acute kidney injury. QJM. 2011;104:237-43.

21. Lapi F, Azoulay L, Yin H, Nessim SJ, Suissa S. Concurrent use of diuretics, angiotensin converting enzyme inhibitors, and angiotensin receptor blockers with non-steroidal anti- 
inflammatory drugs and risk of acute kidney injury: nested case-control study. BMJ. 2013;346:e8525.

22. Bickel M, Khaykin P, Stephan C, Schmidt K, Buettner M, Amann K, et al. Acute kidney injury caused by tenofovir disoproxil fumarate and diclofenac co-administration. HIV Med. 2013;14:633-8.

23. Gandhi S, Fleet JL, Bailey DG, McArthur E, Wald R, Rehman F, et al. Calcium-channel blocker-clarithromycin drug interactions and acute kidney injury. JAMA. 2013;310:254453.

24. Yue Z, Shi J, Jiang P, Sun H. Acute kidney injury during concomitant use of valacyclovir and loxoprofen: detecting drug-drug interactions in a spontaneous reporting system. Pharmacoepidemiol Drug Saf. 2014;

25. Zapletal E, Rodon N, Grabar N, Degoulet P. Methodology of integration of a clinical data warehouse with a clinical information system: the HEGP case. Stud Health Technol Inform. 2010;160:193-7.

26. Boussadi A, Caruba T, Zapletal E, Sabatier B, Durieux P, Degoulet P. A clinical data warehouse-based process for refining medication orders alerts. J Am Med Inform Assoc [Internet]. 2012 [cited 2013 Apr 16]; Available from: http://jamia.bmj.com/content/early/2012/04/19/amiajnl-2012-000850

27. Gaião S, Cruz DN. Baseline creatinine to define acute kidney injury: is there any consensus? Nephrol. Dial. Transplant. 2010;25:3812-4.

28. Pickering JW, Endre ZH. Back-Calculating Baseline Creatinine with MDRD Misclassifies Acute Kidney Injury in the Intensive Care Unit. CJASN. 2010;5:1165-73. 
in HEGP

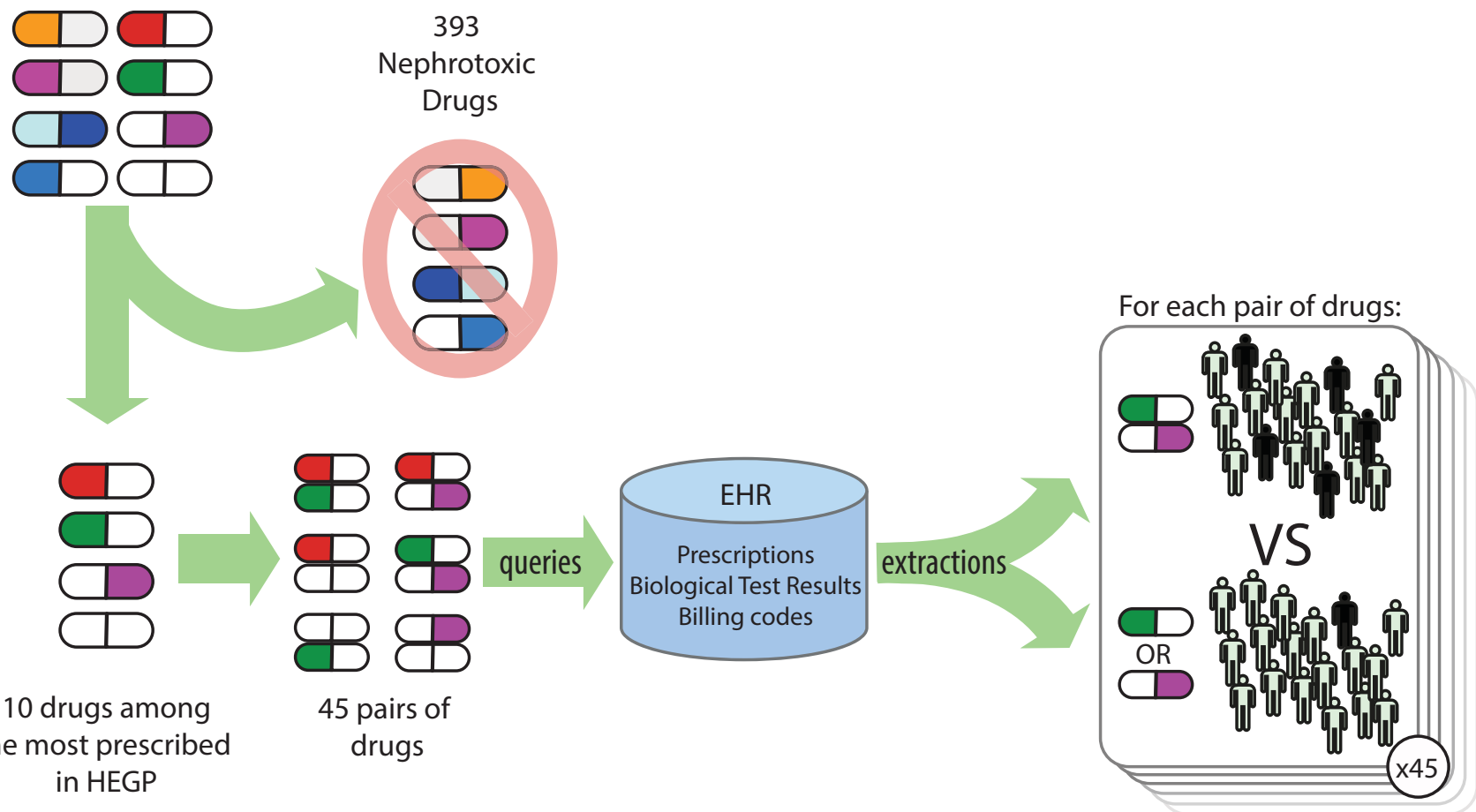



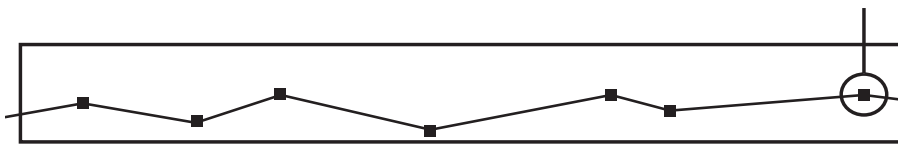
HEGP CDW

In \& Out Patients

$(n=606,524)$

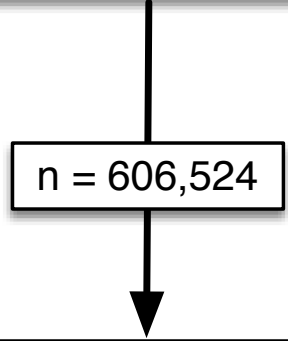

Patients with at least

2 serum creatinine levels

$(n=299,786)$

Patients with at least

1 electronic prescription

$(n=97,590)$

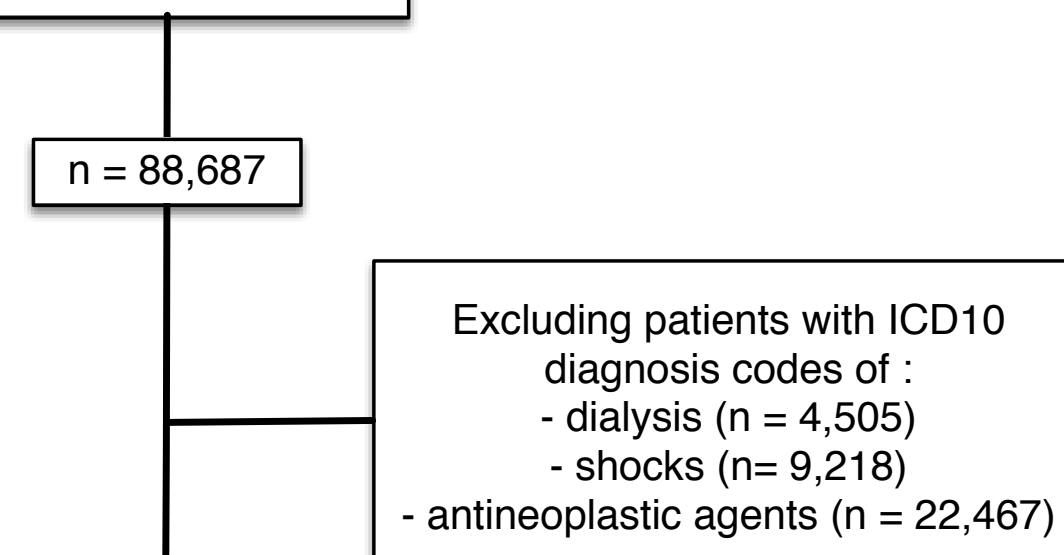

Included Patients

$(n=76,067)$ 